\title{
Dysphagia Evaluation Practices of Speech and Language Therapists in Ireland: Clinical Assessment and Instrumental Examination Decision-Making
}

\author{
Catharine M. Pettigrew and Ciara O’Toole \\ Department of Speech and Hearing Sciences, University College Cork, Cork, Ireland
}

\begin{abstract}
The purpose of this study was to investigate clinical assessment practices and instrumental examination decision-making by speech and language therapists (SLTs) in Ireland. A 21-question survey (including patient scenarios) was sent to 480 SLTs in Ireland. A total of 261 completed surveys were returned (54\%), providing demographic information on SLTs currently working in Ireland and their services. Of these 261 surveys, 70 provided the data for the study, focusing on SLTs currently working in dysphagia, with adults/seniors at least some of the time. The results also showed clinician variability regarding which components are included in a bedside clinical examination of swallowing, with a high degree of consistency for only 11 of the 20 components. Clinicians agreed in their instrumental vs. noninstrumental evaluation recommendations for two of the six patient scenarios, with wide variability in clinical decision-making. Possible influences on clinical decision-making are discussed in relation to the findings of similar previous studies, as well as the current status and future needs of dysphagia training and services in Ireland.
\end{abstract}

Key words: Dysphagia - Bedside evaluation of swallowing - Instrumental assessment - Videofluoroscopy — Speech and language therapy Assessment - Deglutition - Deglutition disorders.

It is well-established that speech and language therapists (SLTs) are the lead experts in communication

Correspondence to: Catharine M. Pettigrew, Department of Speech and Hearing Sciences, University College Cork, 1st Floor Brookfield Health Sciences Complex, Cork, Ireland; E-mail: c.pettigrew@ ucc.ie and swallowing disorders, including the assessment, differential diagnosis, intervention and management of individuals with these disorders [1,2]. With regard to the management of individuals with a swallowing disorder (dysphagia), in particular, inappropriate management can place patients at high risk of aspiration, respiratory infections, choking/death, poor nutrition and weight loss, poor health, anxiety and stress within the family, hospital/admission or extended hospital stay, and reduced quality of life $[1,3$, 4]. The goals of swallowing evaluations are to determine the presence, nature, and cause of the swallowing problem, current level of dysfunction, and nutritional status, and to develop strategies for stabilization and rehabilitation [5]. To achieve these goals it is clearly important that dysphagia evaluation and management practices within the SLT profession are consistent, clear, and well-defined.

Numerous policy statements have been published by several national professional bodies defining the SLT's role in dysphagia management and the requisite knowledge base and skills, policies, and guidelines for intervention, and areas of research [2,6, 7]. According to these policies and guidelines, the SLT's scope of practice includes both the clinical/ bedside examination and instrumental assessment of the oral, pharyngeal, and upper esophageal phases of swallowing function [1]. The clinical bedside examination for dysphagia is a noninstrumental procedure that usually includes gathering information on the current swallowing problem, reviewing medical history, observing signs relevant to the patient's medical status, conducting an examination of speech and swallowing structures, and observing the patient during trial swallows [8]. Typically, the results of this examination will provide the SLT with enough infor- 
mation to decide on the next course of action, for example, whether further instrumental assessment of the problem is necessary, and what type of assessment would be most appropriate $[8,9]$. Instrumental examinations can include videofluoroscopy (VFS) or modified barium swallow, fiberoptic endoscopic evaluation of swallowing (FEES), ultrasonography (ultrasound), manometry, and scintigraphy $[8,10]$. Each of these instrumental procedures has specific advantages and disadvantages [9] and require special training.

It is not unreasonable to suggest that variability in both SLT education/training and the availability of instrumental procedures may influence the consistency with which dysphagia is evaluated by therapists. In addition, much of the research literature on the efficacy of specific diagnostic methods (clinical and instrumental) can be conflicting, possibly contributing to substantial variability in therapist recommendations. A recent survey by MathersSchmidt and Kurlinski [9] found that SLTs in western Washington State differed regarding which components they would include in a clinical examination of swallowing and varied widely in their clinical decision-making based on recommendations for clinical case scenarios (e.g., further instrumental assessment). These authors reported that their findings were comparable with other studies where variability in clinical practice has raised concerns [11, 12].

In light of these findings, it is reasonable to propose that if regions/countries in which the SLT profession has been long-established are experiencing inconsistencies in professional practice, then countries in which the SLT profession is relatively new, and rapidly expanding, may experience particular challenges in this area. The profession of speech and language therapy in the Republic of Ireland has recently been experiencing a period of rapid exponential growth. Furthermore, many overseas graduates are employed in Ireland, resulting in a mix of nationalities and educational experience among SLTs across the country and increasing the services provided to the community.

In response to the rapid growth of the profession, the Irish Association of Speech and Language Therapists (IASLT) is currently in the process of establishing national practice guidelines for SLTs working in Ireland, including guidelines for dysphagia management. Therefore, it is particularly important and timely to investigate the consistency of current SLT dysphagia evaluation practices across the country. To date there has been no such study; therefore, the aim of the current study was to investigate clinical assessment practices and instrumental examination decision-making by SLTs in Ireland.

\section{Methods}

\section{Participants}

In May 2005, a 21-question survey was sent to 480 speech and language therapists in the Republic of Ireland. First, an email was sent to all SLT managers from the IASLT endorsing the survey, with an attached letter from the principal investigator requesting support and participation in the study. The appropriate number of surveys for staff to complete were then forwarded to the managers with a cover letter describing the aims and objectives of the study. A total of 261 completed surveys (54\%) were returned by post. Demographic participant information included years practicing as SLT, patient caseload, job setting, work hours per week, and information on dysphagia training. Following collection of the demographic data, questionnaires were withdrawn from the data pool if the respondents indicated that they had no clinical experience with dysphagia patients within the last 12 months, did not work with adults and/or seniors at least some of the time within their caseload, and/or were not qualified to assess all three of the first three stages of swallowing (oral-preparatory, oral, and pharyngeal). The remaining 70 surveys (27\% of the original total) provided the data on dysphagia services, evaluation practices, and clinical decision-making. Background information on these 70 participants is provided in Table 1.

Over half of the respondents (54.3\%) in the reduced pool of data had one to five years of experience as speech and language therapists. Most respondents (82.8\%) worked 30 or more hours per week, and the majority of the clinicians $(67.1 \%)$ reported that $50 \%$ or more of their caseload in the last 12 months comprised dysphagia assessment and management. Regarding educational background, the majority of respondents $(N=59 / 65,90.1 \%)$ received classroom dysphagia training (lecture format) in their undergraduate courses, and those who did not had at least some theoretical postgraduate training in dysphagia. Accordingly, most of the respondents ( $N=63 / 67,94 \%)$ received some postgraduate classroom dysphagia training. Just over half of the participants $(N=34 / 66,51.5 \%)$ indicated that they had no supervised clinical experience at the undergraduate level, but the majority of respondents $(N=49 / 62,79 \%)$ reported having some supervised clinical experience at the postgraduate level. The similar number of respondents indicating on-the-job experience $(N=53 / 62,85 \%)$ is most likely because these postgraduate and on-the-job clinical hours can represent the same experience for SLTs. Clinicians were trained in a variety of countries across both hemispheres; however, the majority were trained in Ireland at both undergraduate (62.5\%) and postgraduate $(60.6 \%)$ levels.

Given the instrumentation types listed, VFS was the most commonly available procedure ( $N=46 / 70,65.7 \%$ available within facility; $N=60 / 70,90.9 \%$ within 30 miles) (Fig. 1). The next most available procedure was FEES ( $N=26 / 63,41.3 \%$ available within facility; $N=38 / 62,61.3 \%$ within 30 miles). Twenty-two respondents $(31.4 \%)$ from a wide range of job settings indicated that there were no instrumental procedures available at their facilities. Furthermore, $28.6 \%$ of participants $(20 / 70)$ indicated that their recommendations for dysphagia evaluation services were limited by regional access to instrumentation.

\section{Survey}

The survey in this study was adapted from that developed by Mathers-Schmidt and Kurlinski [9], with copyright permission from the original authors. Minor modifications were made to the 
Table 1. Survey participant demographics (overall $N=70$ )

\begin{tabular}{|c|c|c|}
\hline & $N^{\mathrm{a}}$ & $\%$ \\
\hline SLT experience in years & 70 & \\
\hline $1-5$ & 38 & 54.3 \\
\hline $6-10$ & 15 & 21.4 \\
\hline $11-15$ & 10 & 14.3 \\
\hline $16-20$ & 4 & 5.7 \\
\hline$>20$ & 3 & 4.3 \\
\hline Hours worked/week & 70 & \\
\hline $1-9$ & 0 & 0 \\
\hline $10-19$ & 1 & 1.4 \\
\hline $20-29$ & 11 & 15.7 \\
\hline $30-39$ & 54 & 77.1 \\
\hline $40+$ & 4 & 5.7 \\
\hline$\%$ of caseload dysphagia within the last year & 70 & \\
\hline $1-9$ & 6 & 8.6 \\
\hline $10-24$ & 9 & 12.9 \\
\hline $25-49$ & 8 & 11.4 \\
\hline $50-74$ & 21 & 30.0 \\
\hline $75+$ & 26 & 37.1 \\
\hline \multicolumn{3}{|c|}{ Hours of dysphagia training before treating patients (lecture format) } \\
\hline Undergraduate & 65 & \\
\hline None & 6 & 9.2 \\
\hline $1-5$ & 17 & 26.2 \\
\hline $6-10$ & 21 & 32.3 \\
\hline $11-15$ & 10 & 15.4 \\
\hline $16+$ & 11 & 16.9 \\
\hline Postgraduate & 67 & \\
\hline None & 4 & 6.0 \\
\hline $1-10$ & 3 & 4.5 \\
\hline $10-20$ & 2 & 3.0 \\
\hline $20-40$ & 32 & 47.8 \\
\hline $40+$ & 26 & 38.8 \\
\hline \multicolumn{3}{|c|}{ Hours of supervised clinical experience before treating patients } \\
\hline Undergraduate & 66 & \\
\hline None & 34 & 51.5 \\
\hline $1-5$ & 10 & 15.2 \\
\hline $6-10$ & 5 & 7.6 \\
\hline $11-15$ & 6 & 9.1 \\
\hline $16+$ & 11 & 16.7 \\
\hline Postgraduate & 62 & \\
\hline None & 13 & 21.0 \\
\hline$<40$ & 11 & 17.7 \\
\hline $40-60$ & 25 & 40.3 \\
\hline $60+$ & 13 & 21.0 \\
\hline On-the-Job & 62 & \\
\hline None & 9 & 14.5 \\
\hline$<40$ & 18 & 29.0 \\
\hline $40-60$ & 16 & 25.8 \\
\hline $60+$ & 19 & 30.6 \\
\hline Country of undergraduate training & 64 & \\
\hline Ireland & 40 & 62.5 \\
\hline Australia & 6 & 9.4 \\
\hline UK/Britain/Northern Ireland & 14 & 21.9 \\
\hline USA & 2 & 3.1 \\
\hline Netherlands & 1 & 1.6 \\
\hline South Africa & 1 & 1.6 \\
\hline Country of postgraduate/on-the-job training & 66 & \\
\hline Ireland & 40 & 60.6 \\
\hline Australia & 3 & 4.5 \\
\hline UK/Britain/Northern Ireland & 6 & 9.1 \\
\hline
\end{tabular}

Table 1. Continued

\begin{tabular}{lrr}
\hline & $N^{\mathrm{a}}$ & $\%$ \\
\hline USA & 2 & 3.0 \\
$>1$ country & 15 & 22.7 \\
\hline
\end{tabular}

${ }^{\mathrm{a}}$ The total number of participant responses for that question.

original survey to suit Irish participants (e.g., types of job settings, hours of training/clinical experience). In accordance with the recommendations of Mathers-Schmidt and Kurlinski [9], demographic questions were expanded to provide more precise and detailed information on clinician training, including the levels of dysphagia training (undergraduate/postgraduate) and in what countries clinicians were trained.

In addition to requesting demographic information, the survey requested information on the frequency with which SLTs include certain components in a clinical ("bedside") examination for dysphagia. Participants indicated frequency of including a component by circling a corresponding number, 1-5, where $1=$ never, $2=$ seldom, $3=$ half the time, $4=$ usually, $5=$ always. The component "pulse oximetry" was added to those obtained from the original survey. It is a procedure that allows continuous monitoring of arterial oxygen saturation $\left(\mathrm{SpO}_{2}\right)$ and pulse rate by directing red and infrared light through a pulsating vascular bed such as a finger or a toe [13].

The survey also included the six patient scenarios from the original survey, whereby the participants were asked to indicate the next course of action they would recommend in the management of the patient in each scenario. Response options included videofluoroscopic study (modified barium swallow evaluation), fiberoptic endoscopic evaluation of swallowing, ultrasound, pharyngeal manometry/manofluorography, scintigraphy, no instrumental evaluation (and recheck patient as indicated), and other (please specify recommendation). The "other" option in the case scenarios was modified to instruct clinicians to describe specifically what the "other" recommendation involves, as suggested by the authors of the original survey, in order to facilitate a more accurate interpretation of responses. The modifications to the original survey were reviewed for content validity and clarity by Irish dysphagiatrained SLTs at University College Cork, and the Adult Dysphagia Special Interest Group of Ireland. To ensure consistent interpretation of the respondents' answers to the patient scenario questions, the responses were reviewed and the data entry process completed by the first author, and then a second time for those responses from the reduced data pool ( $N=70 ; 420$ patient scenario responses) with $99.5 \%$ agreement. All patient scenario responses from the reduced data pool were reviewed by the second author, with a $98.1 \%$ level of agreement between authors. Discrepancies were discussed and $100 \%$ interjudge agreement was reached.

\section{Analysis}

Similar to Mathers-Schmidt and Kurlinski [9], descriptive statistics were used to summarize background demographic information $(N=70)$, current availability of instrumental procedures, the degree to which specific components are included in a clinical (bedside) swallowing evaluation, and how consistently these components are included. The percentage of responses for each answer option was calculated to determine which components of the clinical examination are most commonly used (never, seldom, half the time, usually, or always). The total number of interpretable 


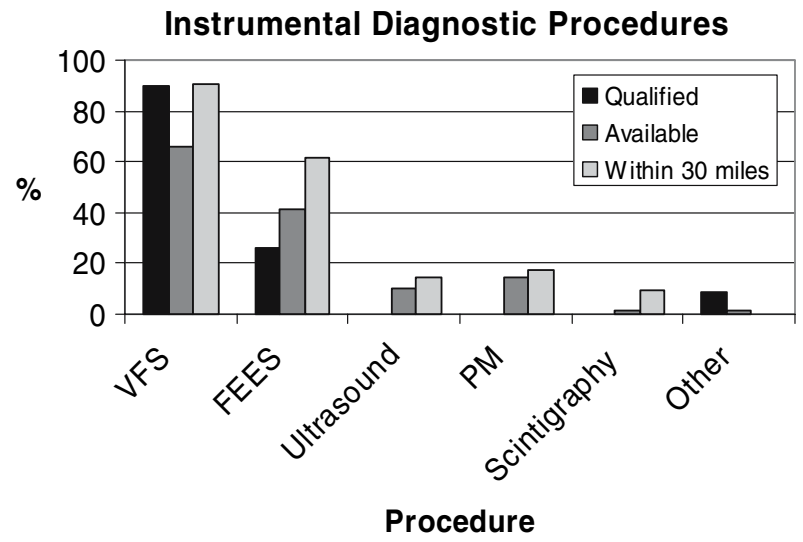

Fig. 1. Information on which instrumental diagnostic procedure(s) respondents were qualified to administer independently or with another professional and the availability of these procedures. "Available" = available at facility; "Within 30 miles" = available within 30 miles of facility. VFS = videofluoroscopy; FEES = fiberoptic endoscopic evaluation of swallowing; PM = pharyngeal manometry.

responses varied across components due to missing data points or multiple options selected for one item. If more than one answer was circled for a component, the response to that item was not included in the data. The "always" and "usually" response options were combined and components were categorized into one of three groups: components always/usually used by $90 \%$ or more of the respondents, components always/usually used by $50 \%-90 \%$ of the respondents, and components always/usually used by less than $50 \%$ of the respondents [9].

The consistency of clinical examination practice was determined by investigating how many components were included in the examination with the same frequency across respondents. Data were categorized as follows: highly consistent, $75 \%$ of respondents indicated the same frequency of component inclusion; moderately consistent, $50 \%-75 \%$ indicated the same frequency of component inclusion; and inconsistent, less than $50 \%$ of respondents indicated the same frequency of component inclusion [9].

Finally, the responses to the patient scenarios were analyzed to determine whether respondents would recommend an instrumental evaluation as the next course of action. The percentage of clinicians choosing each option was determined, then for each scenario response frequencies and percentages were calculated for instrumental versus noninstrumental options. Responses were excluded from analysis if a respondent chose multiple answers and did not clearly indicate what the recommendation for the next course of action would be [9]. (Valid subjects/total subjects per scenario: Scenario 1, 65/70; Scenario 2, 65/70; Scenario 3, 54/70; Scenario 4, 55/70; Scenario 5, 65/70; Scenario 6, 66/70.)

\section{Results}

Components of the Clinical/Bedside Examination for Dysphagia and Consistency of Practices

All of the participants in the reduced data pool $(N=70)$ indicated that they "usually" or "always" conduct a clinical (i.e., "bedside") examination for dysphagia before determining the need for an instrumental diagnostic procedure. The frequencies and percentages of responses indicating how frequently respondents use the listed items in a clinical/ bedside examination are presented in Table 2. Consistency of clinical examination practice across SLTs is also indicated in Table 2, with clinical practice shown to be highly consistent for 11 of the 20 components.

Components were categorized according to combined percentages for "usually" or "always" used and are presented in Table 3.

\section{Consistency of Clinical Decision-Making \\ (Instrumental vs. Noninstrumental)}

The responses to the patient scenarios are summarized in Table 4. For each scenario, the total number of recommendations for instrumental examination and the specific types of instrumentation recommended are shown. Responses in the "other" category for Scenarios 1 and 2 included modified diet/diet change, nil per oral (NPO), referrals (dietitian), and repeated bedside assessments. "Other" responses for Scenario 3 included referrals to an ear, nose, and throat specialist (ENT) or to a gastroenterologist. For Scenario 4, "other" responses included referrals to ENTs, physicians, and/or gastroenterologists, referrals for stroboscopy/digital stroboscopy, and a pH probe. Lastly, "other" responses for Scenario 5 included oromotor exercises, referral to ENT, compensatory strategies, and requests for more information to answer the question adequately.

\section{Analysis of Factors Influencing Clinical Decision-Making}

Similar to Mathers-Schmidt and Kurlinski [9], possible factors influencing clinical decision-making were investigated, including instrumentation availability, dysphagia classroom training, and supervised clinical training. In addition, the country of dysphagia training and qualifications in the use of instrumental diagnostic procedures were also investigated as influencing factors.

First, two categories were created based on the responses to Question 11 regarding availability of instrumental diagnostic procedures: "available" (including the clinicians who indicated that they had access to at least one instrumental diagnostic procedure at their facility, $N=48 ; 68.6 \%$ ), and "none" (including the remaining 22 SLTs; $31.4 \%$ ). The re- 
Table 2. Frequency and percentage of use of clinical examination components

\begin{tabular}{|c|c|c|c|c|c|c|c|c|c|c|c|c|}
\hline \multirow[b]{2}{*}{ Component } & \multirow[b]{2}{*}{$N$} & \multirow[b]{2}{*}{$\mathrm{C}^{\mathrm{a}}$} & \multicolumn{2}{|c|}{ Never } & \multicolumn{2}{|c|}{ Seldom } & \multicolumn{2}{|c|}{$\begin{array}{l}\text { Half the } \\
\text { time }\end{array}$} & \multicolumn{2}{|c|}{ Usually } & \multicolumn{2}{|c|}{ Always } \\
\hline & & & No. & $\%$ & No. & $\%$ & No. & $\%$ & No. & $\%$ & No. & $\%$ \\
\hline Obtain patient history & 69 & $\mathrm{HC}$ & & & & & & & 2 & 2.9 & 67 & 97.1 \\
\hline Patient interview/patient perception of problem & 69 & $\mathrm{MC}$ & & & 1 & 1.4 & & & 17 & 24.6 & 51 & 73.9 \\
\hline Screening/assessment of language abilities & 67 & $\mathrm{MC}$ & 2 & 3.0 & 4 & 6.0 & 9 & 13.4 & 18 & 26.9 & 34 & 48.6 \\
\hline Screening/assessment of mental status & 67 & IC & 6 & 9.0 & 12 & 17.9 & 8 & 11.9 & 16 & 23.9 & 25 & 37.3 \\
\hline Assessment of speech function & 68 & $\mathrm{MC}$ & 1 & 1.5 & 5 & 7.4 & 7 & 10.3 & 17 & 25 & 38 & 55.9 \\
\hline Structural/functional oral motor examination & 69 & $\mathrm{HC}$ & & & & & & & 7 & 10.1 & 62 & 89.9 \\
\hline Adequacy of dentition for chewing & 69 & $\mathrm{HC}$ & & & & & & & 11 & 15.9 & 58 & 84.1 \\
\hline Assessment of sensory function & 68 & IC & 3 & 4.4 & 6 & 8.8 & 11 & 16.2 & 22 & 32.4 & 26 & 38.2 \\
\hline Presence/strength of gag reflex & 68 & IC & 5 & 7.4 & 11 & 16.2 & 7 & 10.3 & 16 & 23.5 & 29 & 42.6 \\
\hline Presence/strength of volitional cough & 69 & $\mathrm{HC}$ & 1 & 1.4 & & & 4 & 5.8 & 7 & 10.1 & 57 & 82.6 \\
\hline Use of a variety of bolus types & 68 & $\mathrm{HC}$ & & & & & 1 & 1.5 & 15 & 22.1 & 52 & 76.5 \\
\hline Adequacy of lip seal & 69 & $\mathrm{HC}$ & & & & & & & 5 & 7.2 & 64 & 92.8 \\
\hline Judgment of efficiency of oral movements & 69 & $\mathrm{HC}$ & & & & & & & 4 & 5.8 & 65 & 94.2 \\
\hline Judgment of pharyngeal delay & 69 & $\mathrm{HC}$ & & & & & & & 6 & 8.7 & 63 & 91.3 \\
\hline Adequacy/strength of laryngeal excursion & 67 & $\mathrm{HC}$ & & & & & 1 & 1.5 & 6 & 9.0 & 60 & 89.6 \\
\hline Assessment of vocal quality (pre/postswallow) & 69 & $\mathrm{HC}$ & & & & & & & 7 & 10.1 & 62 & 89.9 \\
\hline Indirect laryngoscopy & 66 & $\mathrm{HC}$ & 55 & 83.3 & 8 & 12.1 & 1 & 1.5 & & & 2 & 3.0 \\
\hline Cervical auscultation & 68 & IC & 4 & 5.9 & 6 & 8.8 & 5 & 7.4 & 20 & 29.4 & 33 & 48.5 \\
\hline Pulse oximetry & 68 & IC & 2 & 2.9 & 12 & 17.6 & 20 & 29.4 & 20 & 29.4 & 14 & 20.6 \\
\hline Trials with compensatory techniques & 69 & IC & & & 4 & 5.8 & 22 & 31.9 & 31 & 44.9 & 12 & 17.4 \\
\hline
\end{tabular}

${ }^{\mathrm{a}} \mathrm{C}=$ consistency; $\mathrm{HC}=$ highly consistent $\mathrm{MC}=$ moderately consistent; $\mathrm{IC}=$ inconsistent practice across clinicians.

sponses included in the "instrumentation" category were as above (i.e., VFS, FEES, ultrasound, pharyngeal manometry, and scintigraphy). The data were entered into $2 \times 2$ contingency tables, and the relationship between instrumentation availability and clinical decision-making was then determined for each patient scenario. The level of significance of the association between the two variables was measured using chi-squared analysis, and the strength/direction of the association was measured using the gamma test statistic [9]. There was no association between the availability of instrumental diagnostic procedures and recommendations (response choices) in the patient scenarios $(p>0.05)$. Similarly, upon further investigation there was no relationship between the availability of videofluoroscopy and its specific recommendation in any of the patient scenarios $(p>0.05)$, despite being the most available instrumentation procedure and the most popular choice of respondents in their clinical decision-making.

Similar analyses of the relationship between the percentage of total patient caseload involving dysphagia evaluation/management and recommendations in the patient scenarios indicated no significant association between the two variables $(p>0.05)$.

The results of the analyses of the relationship between dysphagia training in the classroom (under-
Table 3. Components included in a clinical examination for dysphagia

\begin{tabular}{lc}
\hline $\begin{array}{l}\text { A. Components usually/always used by }>90 \% \\
\text { of respondents }\end{array}$ & $\%$ \\
Obtain patient history & 100 \\
Structural/functional oral motor examination & 100 \\
Adequacy of dentition for chewing & 100 \\
Adequacy of lip seal & 100 \\
Judgment of efficiency of oral movements & 100 \\
Judgment of pharyngeal delay & 100 \\
Assessment of vocal quality (pre/postswallow) & 100 \\
Use of a variety of bolus types & 98.6 \\
Adequacy/strength of laryngeal excursion & 98.6 \\
Patient interview/patient perception of problem & 98.5 \\
Presence/strength of volitional cough & 92.7 \\
B. Components usually/always used by 50\%-90\% & \\
of respondents & \\
Assessment of speech function & 80.9 \\
Cervical auscultation & 77.9 \\
Screening/assessment of language abilities & 77.6 \\
Assessment of sensory function & 70.6 \\
Presence/strength of gag reflex & 66.1 \\
Trials with compensatory techniques & 62.3 \\
Screening/assessment of mental status & 61.2 \\
Pulse oximetry & 50.0 \\
C. Components usually/always used by < 50\% & \\
of respondents & \\
Indirect laryngoscopy & 3.0 \\
\hline
\end{tabular}

graduate and postgraduate levels) and recommendations in the patient scenarios indicated that for 
Table 4. Patient scenario recommendations

\begin{tabular}{|c|c|c|c|c|}
\hline Scenario & Response & Type $^{\mathrm{a}}$ & Frequency & $\%$ \\
\hline \multirow[t]{6}{*}{1} & \multirow[t]{3}{*}{ Instrumental } & & 41 & 63.1 \\
\hline & & VFS & 39 & 60.0 \\
\hline & & FEES & 2 & 3.1 \\
\hline & \multirow[t]{3}{*}{ Noninstrumental } & & 24 & 36.9 \\
\hline & & Review as indicated & 20 & 30.8 \\
\hline & & Other & 4 & 6.2 \\
\hline \multirow[t]{6}{*}{2} & \multirow[t]{3}{*}{ Instrumental } & & 33 & 50.8 \\
\hline & & VFS & 28 & 43.1 \\
\hline & & FEES & 5 & 7.7 \\
\hline & \multirow[t]{3}{*}{ Noninstrumental } & & 32 & 49.2 \\
\hline & & Review as indicated & 24 & 36.9 \\
\hline & & Other & 8 & 12.3 \\
\hline \multirow[t]{7}{*}{3} & \multirow[t]{4}{*}{ Instrumental } & & 44 & 81.6 \\
\hline & & VFS & 32 & 59.3 \\
\hline & & FEES & 7 & 13.0 \\
\hline & & PM & 5 & 9.3 \\
\hline & \multirow[t]{3}{*}{ Noninstrumental } & & 10 & 18.5 \\
\hline & & Monitor & 2 & 3.7 \\
\hline & & Other & 8 & 14.8 \\
\hline \multirow[t]{8}{*}{4} & \multirow[t]{5}{*}{ Instrumental } & & 23 & 41.8 \\
\hline & & VFS & 3 & 5.5 \\
\hline & & FEES & 18 & 32.7 \\
\hline & & $\mathrm{PM}$ & 1 & 1.8 \\
\hline & & Scintigraphy & 1 & 1.8 \\
\hline & \multirow[t]{3}{*}{ Noninstrumental } & & 32 & 58.2 \\
\hline & & Monitor & 1 & 1.8 \\
\hline & & Other & 31 & 56.4 \\
\hline \multirow[t]{6}{*}{5} & \multirow[t]{3}{*}{ Instrumental } & & 50 & 76.9 \\
\hline & & VFS & 45 & 69.2 \\
\hline & & FEES & 5 & 7.7 \\
\hline & \multirow[t]{3}{*}{ Noninstrumental } & & 15 & 23.1 \\
\hline & & Monitor & 9 & 13.8 \\
\hline & & Other & 6 & 9.2 \\
\hline \multirow[t]{6}{*}{6} & \multirow[t]{3}{*}{ Instrumental } & & 58 & 87.9 \\
\hline & & VFS & 54 & 79.1 \\
\hline & & FEES & 5 & 7.6 \\
\hline & \multirow[t]{3}{*}{ Noninstrumental } & & 8 & 12.1 \\
\hline & & Monitor & 7 & 10.6 \\
\hline & & Other & 1 & 1.5 \\
\hline
\end{tabular}

${ }^{\mathrm{a}}$ VFS = videofluoroscopy; FEES $=$ fiberoptic endoscopic evaluation of swallowing; $\mathrm{PM}=$ pharyngeal manometry.

Patient Scenario 4 there was a significant negative association between hours of undergraduate classroom training (low $=0-10 \mathrm{~h}$; high $=11+\mathrm{h}$ ) and recommendations for the next course of action for the patient (instrumental vs. noninstrumental procedure) $\left(\gamma=-0.576 ; \chi^{2}=4.126, p<0.05\right)$. More specifically, those respondents with more hours of undergraduate classroom training tended to choose noninstrumental options for managing the patient, whereas those respondents with less hours of training tended to choose instrumental options.

The results of the analyses of the relationship between hours of supervised clinical experience in dysphagia (undergraduate and postgraduate/on-thejob levels) and recommendations in the patient scenarios indicated that, although not significant, there was an observable negative association for Patient Scenario 6 between hours of supervised postgraduate/on-the-job clinical experience (low $=0-40 \mathrm{~h}$; high $=40+\mathrm{h}$ ) and recommendations for the next course of action for the patient (instrumental vs. noninstrumental procedure) $\left(\gamma=-1.000 ; \quad \chi^{2}=3.319, \quad p<0.1\right), \quad$ such that respondents with higher hours of supervised experience chose noninstrumental options, and respondents with less hours of experience chose instrumental options for managing the patient.

To investigate the possible influence of the country of training and country of clinical experience on clinical decision-making, the countries in which the respondents were trained were grouped into two categories [Ireland/United Kingdom (UK) and nonIreland/UK] for both classroom training (undergraduate and postgraduate) and clinical experience (undergraduate and postgraduate/on-the-job). Respondents who trained in Ireland and/or the UK were grouped together because of the strong crosslinks between the two in training and clinical experiences. If a respondent indicated that he/she had at least some training/clinical experience in a country other than Ireland or the UK, he/she was included in the non-Ireland/UK category. The results indicated that there is no association between the country of training or clinical experience and recommendations (instrumental vs. non-instrumental) in the patient scenarios $(p>0.05)$. Despite the insignificance of this association, further descriptive analysis of the data indicated some observable trends. For example, VFS was the most commonly recommended procedure in most of the patient scenarios, particularly Scenarios 1, 5, and 6. In Patient Scenario 1, 80\% (4/5) of respondents who had received at least some undergraduate classroom training in Australia recommended VFS compared with 59\% (32/54) of those trained in Ireland or the UK. Similarly, in Patient Scenarios 5 and $6,80 \%(4 / 5)$ and $100 \%(6 / 6)$ of Australian-trained respondents recommended VFS, respectively, compared with $68.5 \%$ (37/54) and $77.8 \%$ (42/54) of Irish/UK-trained respondents, respectively. The Canadian-trained respondent (1/1) indicated VFS as his/her recommended procedure for each of the patient scenarios to which he/she responded. Respondents trained in South Africa, USA, and the Netherlands were more mixed in their responses. Interestingly, apart from Patient Scenario 6, the results from the data on country of clinical experience were more consistent across the different countries. In 
Patient Scenario 6, 100\% of respondents who had supervised clinical experience in Australia chose VFS as the recommended procedure compared with $77.8 \%$ of those with experience from Ireland or the UK.

Lastly, respondents' choices in the patient scenarios were analyzed in relation to whether the respondents were qualified to administer the instrumental diagnostic procedures provided as options (i.e., VFS, FEES, pharyngeal manometry, ultrasound, and scintigraphy). The results indicated that there is generally no association between the two variables $(p>0.05)$. However, the results for Patient Scenario 3 show that there is a positive association between the two variables $\left(\gamma=0.745 ; \chi^{2}=5.233\right.$; $p<0.05$ ), such that the qualified respondents (i.e., respondents qualified to administer at least one of the instrumental diagnostic procedures) tended to choose instrumental options as the next course of action, and the nonqualified respondents (i.e., respondents not qualified to administer any instrumental diagnostic procedures) tended to choose noninstrumental options.

\section{Discussion}

The results of the current study indicated that the assessment and management of dysphagia comprises over $50 \%$ of the caseload of the majority of clinicians working in adult dysphagia. This result is consistent with the findings of Code and Heron [14], who reported that on average SLTs working in adults services in the United Kingdom spend nearly 53\% of their time working with dysphagia, with significantly less time spent on communication disorders (e.g., aphasia). More recently, however, it has been reported that the dysphagic caseload has steadily increased over the last decade [15], taking up to $70 \%$ of a significant number of therapists' caseloads in the United Kingdom [16]. Together, these findings highlight the importance of dysphagia management in the role of the SLT and thus the need for consistent evaluation practices across clinicians.

\section{Clinical Examination Components Used and Consistency Across Clinicians}

The results of this study are similar to the findings of Mathers-Schmidt and Kurlinski [9] in that clinicians appear to differ regarding the components they include in a bedside/clinical examination of swallowing. Just over half $(11 / 20)$ of the components were usually or always used by the majority of respondents, and a further component (indirect laryngoscopy) was never or seldom used by the majority of respondents (95.4\%). Again, similar to Mathers-Schmidt and Kurlinski [9], the degree of clinical inconsistency in the components included in the bedside examination was higher than expected. For example, clinical practice is only moderately consistent for patient interview/patient perception of the problem, screening assessment of language abilities, and screening assessment of speech function. This result is not surprising given some discrepancies between the published clinical guidelines of different national bodies. In particular, the clinical guidelines published by the Royal College of Speech and Language Therapists (RCSLT) [6] do not include these three components as specific aspects of assessment to consider in clinical evaluation, yet clinical guidelines published by Speech Pathology Australia [2] and the American Speech-Language-Hearing Association [7] indicate that communication status (including comprehension, hearing speech, and language) is to be assessed in a bedside evaluation whenever applicable. Mathers-Schmidt and Kurlinski [9] also reported only moderate consistency in clinical practice for screening assessments of speech and language function, yet the clinicians in their study (conducted in western Washington State) were highly consistent in their inclusion of a patient interview, in contrast to the clinicians in the current study.

The results of this study also indicated that clinical practice is inconsistent among SLTs in Ireland with respect to the inclusion of screening assessments of mental status and sensory function, presence/strength of gag reflex, cervical auscultation, pulse oximetry, and trials with compensatory techniques, similar to the results of the study by MathersSchmidt and Kurlinski [9]. The inconsistency in the practice of assessment of mental status is unexpected given that clinical guidelines emphasize the need to consider cognitive levels and level of alertness when carrying out a bedside swallowing evaluation $[2,6,7]$. It is possible, however, that many respondents interpreted the component as being a more formal assessment of cognitive/mental status rather than informal observations of cognitive levels and alertness.

According to Logemann [8], oral sensitivity examination (including an assessment of light touch) should be included in the bedside swallow evaluation; however, there are no clear guidelines for interpretation of oral sensitivity testing. This lack of clear interpretation guidelines may be a contributing factor in the inconsistent use of sensory function assessment by clinicians in the current study and in the study by 
Mathers-Schmidt and Kurlinski [9]. Although clinical guidelines from Australia report that clinicians should comment on sensation in clinical oropharyngeal assessments [2], the assessment of sensory function is less explicit in other guidelines [6].

The relatively high percentage of SLTs who still include the presence/strength of the gag reflex component $(66.1 \%)$ and the inconsistency in its exclusion from the bedside evaluation is unexpected given that it is not included in published clinical guidelines $[2,6]$ and there are no data to support the relationship between the presence/absence of a gag reflex in neurologically impaired patients and their ability to swallow [8]. The inconsistency in the use of pulse oximetry and cervical auscultation as components of the bedside evaluation is not as surprising given the conflicting findings in the literature regarding their effectiveness $[8,13,17-20]$. Clinical guidelines and some studies in the literature tend to agree, however, that if used in conjunction with a bedside evaluation, pulse oximetry and cervical auscultation may provide additional information relevant to swallowing function but they cannot be used as stand-alone diagnostic tools $[6,19,21]$. Despite the inconsistent use of cervical auscultation, the results of this study indicated that $50 \%$ of clinicians in Ireland include this component usually/all the time in the bedside swallow evaluation, a higher percentage than reported in previous studies $[9,11]$. Upon further investigation, there was no association found between the inclusion of cervical auscultation in the bedside swallow evaluation and availability of VFS, either at the facility or within 30 miles $(p>0.05)$. Finally, the inconsistent inclusion of compensatory strategy trials in the bedside swallow evaluation may simply be because trials may not be appropriate for every patient and are often undertaken as part of an imaging assessment instead [8].

\section{Clinical Decision-Making}

Overall, respondents varied widely in their clinical decision-making, with the results being strikingly similar to those reported by Mathers-Schmidt and Kurlinski [9] for each of the patient scenarios. Clinicians in the current study showed strong agreement in their recommendations for the next course of action in only two of the patient scenarios, 3 and 6 . In these cases instrumental evaluation was recommended by $81.6 \%$ and $87.9 \%$ of clinicians, respectively. As suggested by Mathers-Schmidt and Kurlinski [9], it is likely that the recommendation for instrumental evaluation in Patient Scenario 3 was prompted by an awareness of possible esophageal obstruction because most respondents who indicated noninstrumental action recommended onward referral to an ENT or gastroenterologist. Patient Scenario 6 included information from previously administered clinical examinations and indicated the development of aspiration pneumonia despite good progress previously. Aspiration is caused by the entry of food or liquid into the airway below the true vocal folds [8]. It is well-documented in the literature that a profound limitation of the traditional bedside examination is its inability to reliably detect silent aspiration (when a patient aspirates with no outward clinical sign such as coughing/choking), because the pharyngeal phase of the swallow is not objectively assessed [4, 22]. As mentioned previously, the result of a bedside screening examination generally helps the SLT to determine whether further, more objective instrumental assessment is warranted [22, 23], so it is not surprising that respondents showed a high-percentage agreement in recommending an instrumental course of action $(87.9 \%)$. It is also not surprising that the diagnostic procedures specified were either VFS $(79.1 \%)$ or FEES $(7.6 \%)$ as these are the two procedures most documented in the literature as being able to reliably detect silent aspiration [24, 25]. Furthermore, clinical guidelines recommend that when information on the presence and cause of aspiration and residue is required to supplement clinical decision-making, VFS or FEES will be performed [6].

Respondents were moderately consistent in their recommendation of instrumental evaluation in Patient Scenario 5 (76.9\%), even though it could be argued that an instrumental evaluation may not be required (and therefore not cost-effective) given the information provided in this scenario [9]. The respondents were fairly evenly split in their recommendations of instrumental vs. noninstrumental evaluations in Patient Scenario 2 and slightly less so for Patient Scenario 1. The one scenario that did not elicit an instrumental evaluation from the majority of respondents was Patient Scenario 4. Of the "noninstrumental" responses, only one participant indicated "no instrumental evaluation" as the next course of action. The remaining 31 participants indicated that the patient should be referred to a specialist (e.g., general practitioner, ENT, or gastroenterologist).

Overall, $67.3 \%$ of the respondents recommended an instrumental assessment as the next course of action in the patient scenarios, a result remarkably similar to the $67.1 \%$ of respondents in the study by Mathers-Schmidt and Kurlinski [9]. According to Mathers-Schmidt and Kurlinski [9], clinicians may be influenced by research studies that highlight the profound limitation of the traditional 
bedside examination's inability to reliably detect silent aspiration $[4,22]$. In contrast to the respondents in the study by Mathers-Schmidt and Kurlinski [9], however, all of the respondents to the patient scenarios in the current study indicated that they usually or always conduct a clinical/bedside evaluation before determining the need for an instrumental diagnostic procedure. Given that a clinical/bedside examination had already been performed in each of the patient scenarios, it is therefore not surprising that more instrumental than noninstrumental assessments were recommended as the next course of action in most of the scenarios.

Overall, the most frequently recommended instrumental evaluation was VFS $(80.7 \%$ of instrumental recommendations, similar to the $86.3 \%$ reported by Mathers-Schmidt and Kurlinski [9]), which is not unexpected given that knowledge of the application and limitations of and suitability for videofluoroscopic evaluation is regarded as a basic for competence as an SLT [2]. It is reasonable to expect that clinicians are most likely to recommend instrumental assessment when equipment is readily available; however, the results of the current study and that by Mathers-Schmidt and Kurlinski [9] indicated that instrumentation availability does not influence clinical decision-making, not even for VFS specifically.

The results of the current study also indicated that factors such as the percentage total patient caseload involving dysphagia evaluation/management, qualifications in instrumental procedure administration, and country of dysphagia training/ experience generally had no influence on clinical decision-making. However, further observations of the data highlighted a possible tendency for clinicians qualified in instrumental procedure administration to recommend such procedures, as well as a possible tendency for Australian- and Canadian-trained clinicians to choose VFS more often than those trained in Ireland or the UK.

Furthermore, hours of dysphagia training and clinical experience at undergraduate and postgraduate/on-the-job levels did not generally influence decision-making, with minor exceptions. In Patient Scenario 4 clinicians with more hours of undergraduate classroom training tended to choose noninstrumental options as the next course of action for the patient (i.e., referral to a specialist), as opposed to the instrumental options chosen by respondents with less hours of training. Similarly, there was a marginally significant association in Patient Scenario 6 such that despite the high percentage of respondents $(87.9 \%)$ recommending VFS as the next course of action, clinicians with more hours of supervised postgraduate/ on-the-job experience chose noninstrumental options in the first instance. More specifically, the cautious approach recommended by these respondents included (a) ensuring there had been no compliance issues or an extension of the CVA before choosing VFS as the next option, (b) deciding on VFS only if indicated by a review bedside assessment performed first, (c) discussing with the team (e.g., nurses) the changes in the patient's status, reviewing the swallow, and proceeding cautiously, and (d) first checking that the modified diet had been adhered to before recommending a VFS. These results may suggest that clinicians who are more highly trained in the classroom at the undergraduate level and who have more supervised clinical experience at the postgraduate level or on-the-job are less likely to recommend instrumental evaluations prematurely or inappropriately. In further support of this finding, a marginally significant relationship between dysphagia experience and recommendation was reported by MathersSchmidt and Kurlinski [9], whereby in Patient Scenario 5 more experienced clinicians tended to recommend no instrumental evaluation and the less experienced clinicians tended to recommend VFS. It is reasonable to propose that clinicians with more training/experience are more confident in their own skills and abilities and therefore less likely to rely on instrumental measurements.

A limitation of the current study was that several respondents indicated more than one option in the patient scenarios or wrote long responses in the "Other" section that involved several sequential steps of action. Many respondents also indicated one option but would make additional comments, for example, that the option chosen would depend on other factors. Other respondents indicated that they were unable to make a decision without further information. These findings perhaps highlight the limitation of using "multiple choice" patient scenarios to accurately measure consistency in clinical decision-making by speech and language therapists, a process that can arguably be fairly subjective and dependent on many different factors.

\section{Conclusion}

The findings of clinician variability in conducting clinical examinations and clinical decision-making are remarkably consistent with those of the study by Mathers-Schmidt and Kurlinski [9], indicating that clinician disagreement in the profession of speech and language therapy is an international concern. However, it is reasonable to expect at this point in time 
that SLTs in Ireland would demonstrate some variability in dysphagia practices given that prevailing national standards are still in the process of being established. It may be that as clinicians become aware of these guidelines following their publication, consistency in clinical practice will increase. As mentioned previously, to date the practice of dysphagia management is a postgraduate level qualification in Ireland and has not been extensively focused on in undergraduate courses. However, in the near future this area of SLT practice is likely to become an undergraduate qualification in Ireland. Accordingly, alongside the published guidelines, the information provided in this study is particularly relevant at this time because it will help Irish educators of SLT students and SLT managers ensure that the knowledge and skill competencies expected of qualified professionals are being achieved.

\section{References}

1. Royal College of Speech \& Language Therapists (RCSLT): Communicating Quality 3. London: Royal College of Speech and Language Therapists, 2006

2. Speech Pathology Australia (SPA): Dysphagia: General. Melbourne: Speech Pathology Australia, 2004

3. Nilsson H, Ekberg O, Olsson R, Hindfelt B: Dysphagia in stroke: a prospective study on quantitative aspects of swallowing in dysphagic patients. Dysphagia 13:32-38, 1998

4. Leder SB, Espinosa JF: Aspiration risk after acute stroke: comparison of clinical examination and fiberoptic endoscopic evaluation of swallowing. Dysphagia 17:214-218, 2002

5. Broniatowski M, Sonies BC, Rubin JS, Bradshaw CR, Spiegel JR, Bastian RW, Kelly JH: Current evaluation and treatment of patients with swallowing disorders. Otolaryngol Head Neck Surg 120:464-473, 1999

6. Royal College of Speech \& Language Therapists (RCSLT): RCSLT Clinical Guidelines. Brackley, UK: Speechmark Publishing, 2005

7. American Speech-Language-Hearing Association (ASHA): Preferred practice patterns for the profession of speech-language pathology. Rockville, MD: ASHA, 1997

8. Logemann JA: Evaluation and Treatment of Swallowing Disorders. 2nd edn. Austin, TX: Pro-Ed, 1998

9. Mathers-Schmidt BA, Kurlinski M: Dysphagia evaluation practices: Inconsistencies in clinical assessment and instrumental examination decision-making. Dysphagia 18:114-125, 2003
10. American Speech-Language Hearing Association (ASHA): Knowledge and skills needed by speech-language pathologists providing services to dysphagic patients/clients. $A S H A$ 32:7-12, 1990

11. McCullough GH, Wertz RT, Rosenbek JC, et al.: Clinicians' preferences and practices in conducting clinical/bedside and videofluoroscopic swallowing examination in an adult, neurogenic population. Am J Speech Lang Pathol 8:149-163, 1999

12. Wilcox F, Liss JM, Siegel GM: Interjudge agreement in videofluoroscopic studies of swallowing. $J$ Speech Hear Res 39:144-152, 1996

13. Colodny N: Comparison of dysphagics and non-dysphagics on pulse oximetry during oral feeding. Dysphagia 15:68-73, 2000

14. Code C, Heron C: Services for aphasia, other acquired adult neurogenic communication and swallowing disorders in the United Kingdom, 2000. Disabil Rehabil 25:1231-1237, 2003

15. Armstrong E: Communication culture in acute speech pathology settings: current issues. Adv Speech Lang Pathol 5:137-143, 2003

16. Rossiter D, Marks L: Reflecting reality. RCSLT Bull April:13-14, 2004

17. Cichero JA, Murdoch BE: The physiologic cause of swallowing sounds: answers from heart sounds and vocal tract acoustics. Dysphagia 13:39-52, 1998

18. Sellars C, Dunnet C, Carter RL: A preliminary comparison of videofluoroscopy of swallow and pulse oximetry in the identification of aspiration in dysphagic patients. Dysphagia 13:82-86, 1998

19. Smith HA, Lee SH, O'Neill PA, Connolly MJ: The combination of bedside swallowing assessment and oxygen saturation monitoring of swallowing in acute stroke: a safe and humane screening tool. Age Ageing 29:495-499, 2000

20. Zenner PM, Losinski DS, Mills RH: Using cervical auscultation in the clinical dysphagia examination in long-term care. Dysphagia 10:27-31, 1995

21. Hembree AC: Dysphagia evaluation and treatment. Operative Tech Otolaryngol Head Neck Surg 8:185-190, 1997

22. Ramsey DJ, Smithard DG, Kalra L: Early assessments of dysphagia and aspiration risk in acute stroke patients. Stroke 34:1252-1257, 2003

23. Rosenbek JC, McCullough GH, Wertz RT: Is the information about a test important? Applying the methods of evidence-based medicine to the clinical examination of swallowing. J Commun Disord 37:437-450, 2004

24. Langmore SE, Schatz K, Olson N: Endoscopic and videofluoroscopic evaluations of swallowing and aspiration. Ann Otol Rhinol Laryngol 100:678-681, 1991

25. Lundy DS, Smith C, Colangelo L, Sullivan PA, Logemann JA, Lazarus CL, Newman LA, Murray T, Lombard L, Gaziano J: Aspiration: cause and implications. Otolaryngol Head Neck Surg 120:474-478, 1999 